\title{
ASTERÁCEAS COMUNES DE LA PROVINCIA DE CANDARAVE- DEPARTAMENTO DE TACNA Y SU IMPORTANCIA COMO RECURSO NATURAL
}

\author{
Juan Franco León'; Liduvina Sulca Quispe ${ }^{2}$ \\ César Cáceres Musaja ${ }^{3}$
}

\section{$R E S U M E N$}

El presente trabajo de investigación tiene como objetivo principal el de recolectar los grupos Taxonómicos (Familias) de nuestra serranía por zonas, las que formarán parte del Herbario "Takana" de nuestra Universidad.

La provincia de Candarave está ubicada a 3,415 m.s.n.m. constituida por 6 distritos, a ocho horas de la ciudad de Tacna. Las recolecciones se realizaron entre los meses de enero a julio de 2000. Se empleó el método preferencial-selectivo considerando la Flora representativa sobre la base de criterios subjetivos. Las muestras son sometidos a prensado, desecación, montaje e identificación.

Los resultados muestran a la Familia Asteraceae mejor representada con 23 especies, entre las mas comunes y utilizadas por el poblador de Candarave.

Entre las especies que predominan están: Ambrosia artemisioides, Baccharis spp, Grindelia tarapacana, Chersodoma jodopappa y Parastrephia quadrangularis.

\section{ABSTRACT}

The present work of investigation has as main objective the one of gathering the groups Taxonómicos (Families) of our serranía for areas, those that will be part of the herbarium "Takana" of our University.

The County of Candarave is located 3,415 m.a.s.l. constituted by six districts, at eight hours from the city of Tacna. The gatherings were carried out among the months of January to Julio 2000. You uses the preferential-selective method considering the representative Flora on the base of subjective approaches. The samples are subjected had pressed, drying, assembly and identification.

The results show to the Family better Asteraceae represented with 23 species, among those but common and used by the resident of Candarave.

Among the species that prevail they are: Ambrosia artemisioides, Baccharis spp, Grindelia tarapacana, Chersodoma jodopappa and Parastrephia quadrangularis. 


\section{INTRODUCCIÓN}

La Familia Asteráceae en el departamento de Tacna se encuentra muy poco estudiada y no hay reportes de las especies existentes.

La Familia Asteráceae se caracteriza fundamentalmente por poseer inflorescencias de cabezuelas con involucros (Capítulos) de uno a muchas flores, sobre un receptáculo común rodeado por filarias; corola unidad con 5 lóbulos; 5 estambres formando un cilindro alrededor del estilo; ovario con 2 ramas del estilo y unilocular con un solo óvulo. Esta familia presenta hierbas y arbustos, con hojas alternas, opuestas o raramente verticilada. Es una de las familias más grandes dentro de las plantas con flores.

La provincia de Candarave se encuentra ubicada al noroeste de Tacna a $164 \mathrm{Km}$, a $70^{\circ} 12^{\prime} 12^{\prime \prime}$ de longitud oeste y $17^{\circ} 15^{\prime} 30^{\prime \prime}$ latitud sur, a una altura de 3415 m.s.n.m. Dicha provincia presenta una topografía variada con valles, cerros, pampas y quebradas que se extienden desde aproximadamente de los 2080 a 5000 m.s.n.m.

Con la finalidad de contribuir al conocimiento de las especies comunes que presenta la Familia Asteráceae en la provincia de Candarave, se ha realizado el presente trabajo. Dentro de los objetivos planteados está el de realizar un estudio taxonómico y utilidades de las especies propias de esta zona.

\section{MATERIALES Y MÉTODOS}

Se realizaron muestreos durante los meses de enero a julio de 1999, abarcando toda la provincia. Se colectaron las muestras utilizando el método preferencial selectivo considerando las especies típicas o representativas, sobre la base de criterios subjetivos. Se utilizó una ficha de campo anotando los datos más importantes como son los aspectos morfológicos y ecológicos. El prensado se realizó en el mismo campo utilizando periódicos, las muestras fueron colocadas una detrás de otra formando una pila de plantas puestas en prensadores de madera. Se realizo el cambió de papel periódico por un lapso de 7 dias y se dejó en la prensadora por un tiempo de 30 días para su total desecación de las muestras. Luego se procedió a realizar el montaje de las muestras en cartulinas blancas de tamaño $27 \times 42$ $\mathrm{cm}$. Para la identificación de las muestras se utilizó bibliografia especializada.

\section{RESULTADOS}

La ubicación taxonómica de las especies en estudio según Croquis 1981.

DIVISIÓNMAGNOLIOPHYTA

\section{CLASE MAGNOLIOPSIDA}

SUBCLASE MAGNOLIIDAE

ORDEN ASTERALES

FAMILIAASTERACEAE

GÉNERO Acanthoxanthium

ESPECIEAcanthoxanthium spinosum (fig. 23)

GÉNERO Ambrosia

ESPECIE Ambrosia artemisioides (fig. 2) Ambrosia arborescens (fig. 1)

GÉNERO Baccharis

ESPECIE Baccharis boliviensis (fig. 14)

Baccharis incarum (fig. 10)

Baccharis sp. (fig. 3)

GÉNERO Bidens

ESPECIE Bidens triplinervia (fig. 4)

GÉNERO Diplostephium

ESPECIE Diplostephium meyenii (fig. 5)

GÉNERO Gnaphalium

ESPECIE Gnaphalium dombeyanum (fig. 7)

Gnaphalium sp. (fig. 6)

GÉNERO Chersodoma

ESPECIE Chersodoma jodopappa (fig. 8)

GÉNERO Grindelia

ESPECIE Grindelia tarapacana (fig. 9)

GÉNERO Matricaria

ESPECIE Matricaria recutita (fig. 11) 
GÉNERO Mutisia

ESPECIE Mutisia acuminata (fig. 12)

GÉNERO Ophryosporus

ESPECIE Ophryosporus peruvianus(fig.13)

GÉNERO Parastrephia

ESPECIE Parastrephia quadrangularis (fig.15)

\section{GÉNERO Perezia}

ESPECIE Perezia multiflora (fig. 16)

GÉNERO Proustia

ESPECIE Proustia berberidifolia (fig. 17)

\section{GÉNERO Senecio}

ESPECIE Senecio sp. (fig. 18)

GÉNERO Tagetes

ESPECIE Tagetes multiflora (fig. 19)

GÉNERO Taraxacum

ESPECIE Taraxacum officinale (fig. 20)

\section{GÉNERO Tessaria}

\section{ESPECIE Tessaria integrifolia (fig. 21)}

reportarlas para esta zona sur andina del Perú.

\section{REFERENCIAS BIBLIOGRÁFICAS}

CRONQUIST, A. (1981). An Integral System of clasification of flowering plants. Columbia University Press, Nueva York.

FERREYRA, R. (1986). Flora del Perú: Dicotiledoneas. Ed. U.N.M.S.M., Lima-Perú.

MOSTACERO, J. y MEJíA, F. (1993). Taxonomia de las Fanerógamas Peruanas. CONCYTEC. Trujillo-Perú.

REYNEL, C. y LEÓN, J. (1990). Arboles y arbustos andinos para la Agroforesteria y Conservación de Suelos. Ed. Ministerio de Agricultura: Dirección General Forestal y Fauna. Lima-Perú.

\section{GÉNERO Viguiera}

\section{ESPECIE Viguiera procumbens (fig. 22)}

\section{DISCUSIÓN Y CONCLUSIONES}

Son muchas las asteráceas encontradas hasta el mes de julio en la provincia de Candarave, algunas comportándose como invasoras de cultivos diversos, otras como plantas silvestres, y tambien muchas de ellas son empleadas en el tratamiento de enfermedades y como combustible para la elaboración de alimentos. Entre las especies medicinales se puede considerar a Proustia berberidifolia "Taza", utilizada para la cura de los bronquios; Baccharis sp. "chare" y Bidens triplinervia "Misico", utilizada para los dolores estomacales; Glindelia tarapacana "chiñe". muy utilizada para aliviar los dolores musculares entre otras. En la sierra se le aprovecha, por ejemplo, las Baccharis sp. y Tessaria integrifolia son exelentes potencialidades en agroforesteria, es decir, para la conservación de suelos porque su sistema radicular se ramifica densamente y no es demasiado largo, de modo que no ofrece competencia a los cultivos y tiene gran facilidad de propagación natural asimismo presenta alta tolerancia al clima de la zona y sequías.

Las tolas Chersodoma jodopappa y Parastrephia quadrangularis forman extensas comunidades (tolares) y son empleadas como combustible.

Asimismo, se puede concluir que son 23 especies de asteraceas identificadas hasta el momento, faltando aún muchas todavia por catalogarlas y

RODRIGUES, D. (1999). Estudio de la biodiversidad: Cuenca del Cotahuasi. Ed. Akuarella. Arequipa-Perú.

SAGÁSTEGUI, A. y LEIVA, G. (1993). Flora invasora de los cultivos del Perú. CONCYTEC. Trujillo-Perú.

SOUKUP, J. (1970). Vocabulario de ios Nombres Vulgares de la Flora Peruana. Lima-Perú.

TOVAR, O. (1953). Revisión de las especies del género Perezia. Publicación Mus. Hist. Nat. Serie B. Bot. N ${ }^{0} 8$.

WEBERBAVER, A. (1945). El mundo vegetal de los andes peruanos. Est. Exp. La Molina. Ministerio de Agricultura. 

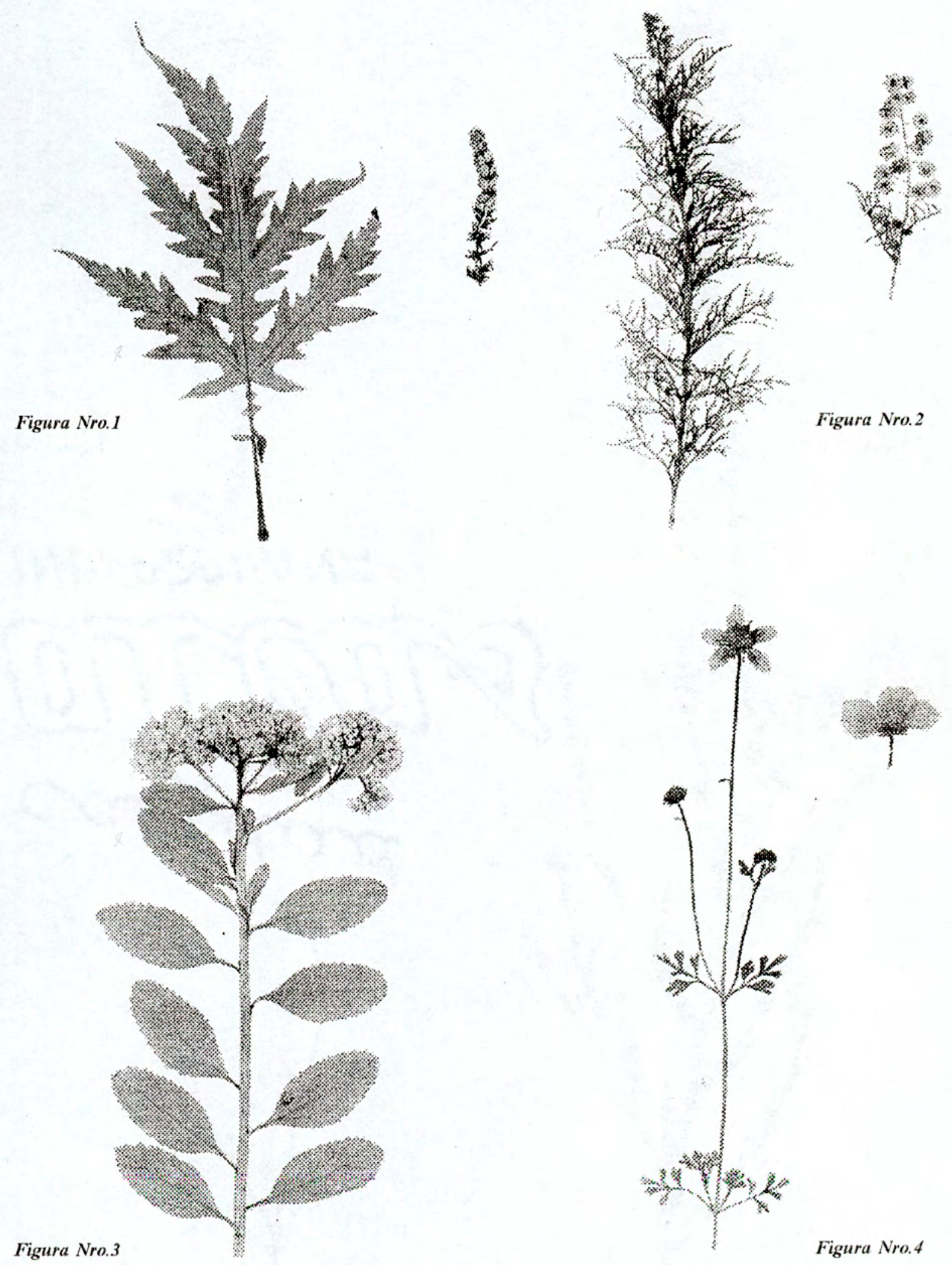
Ciencia \& Desarrollo 7
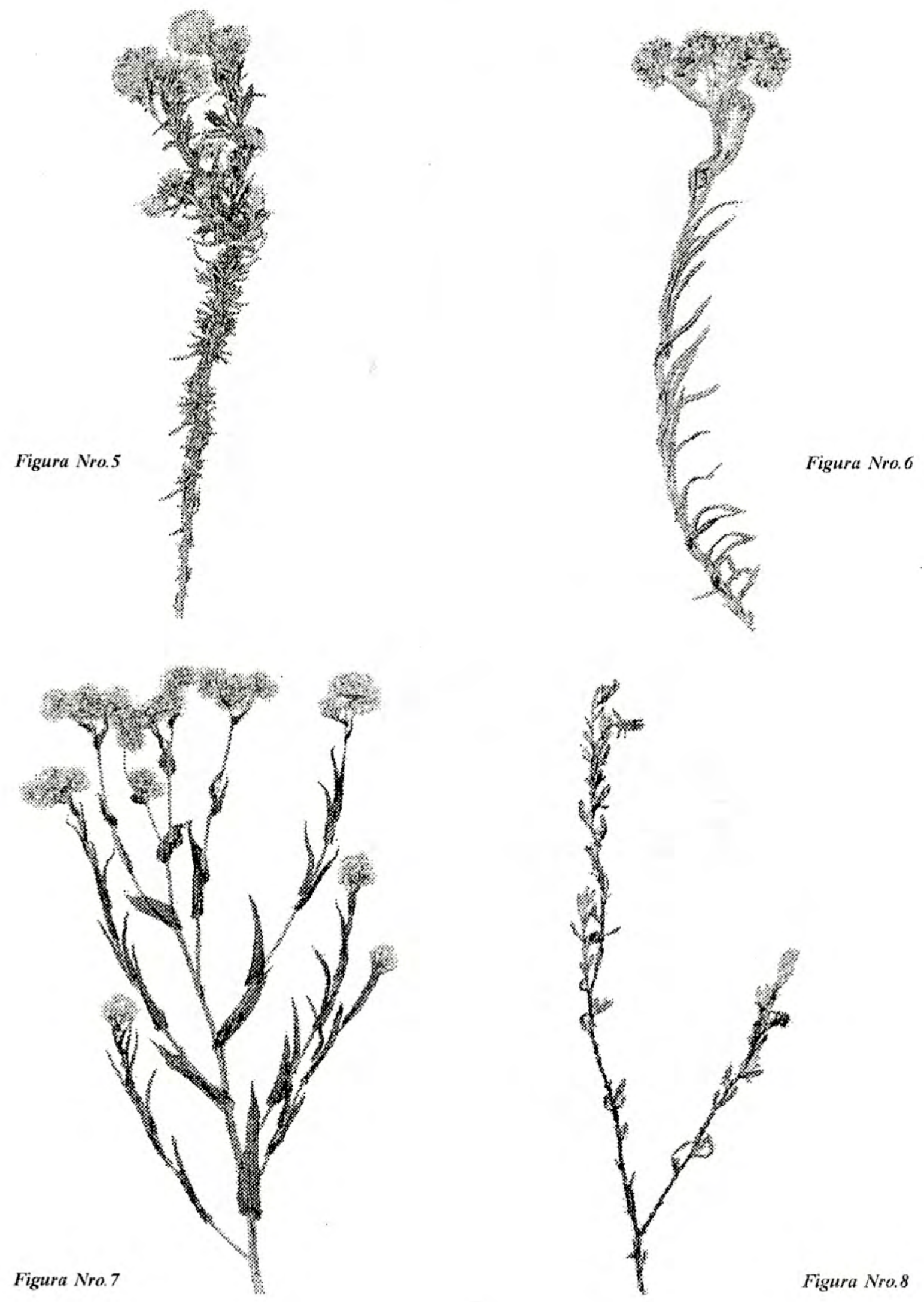

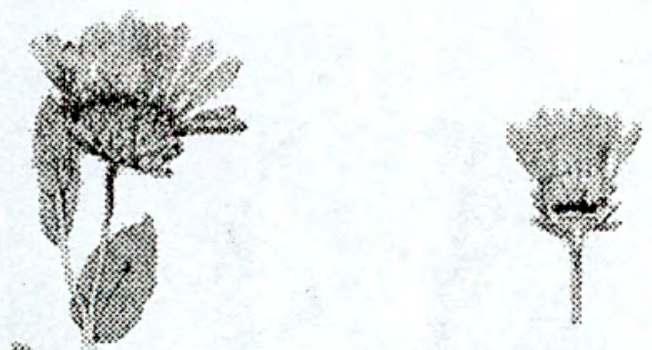

Figura Nro.9
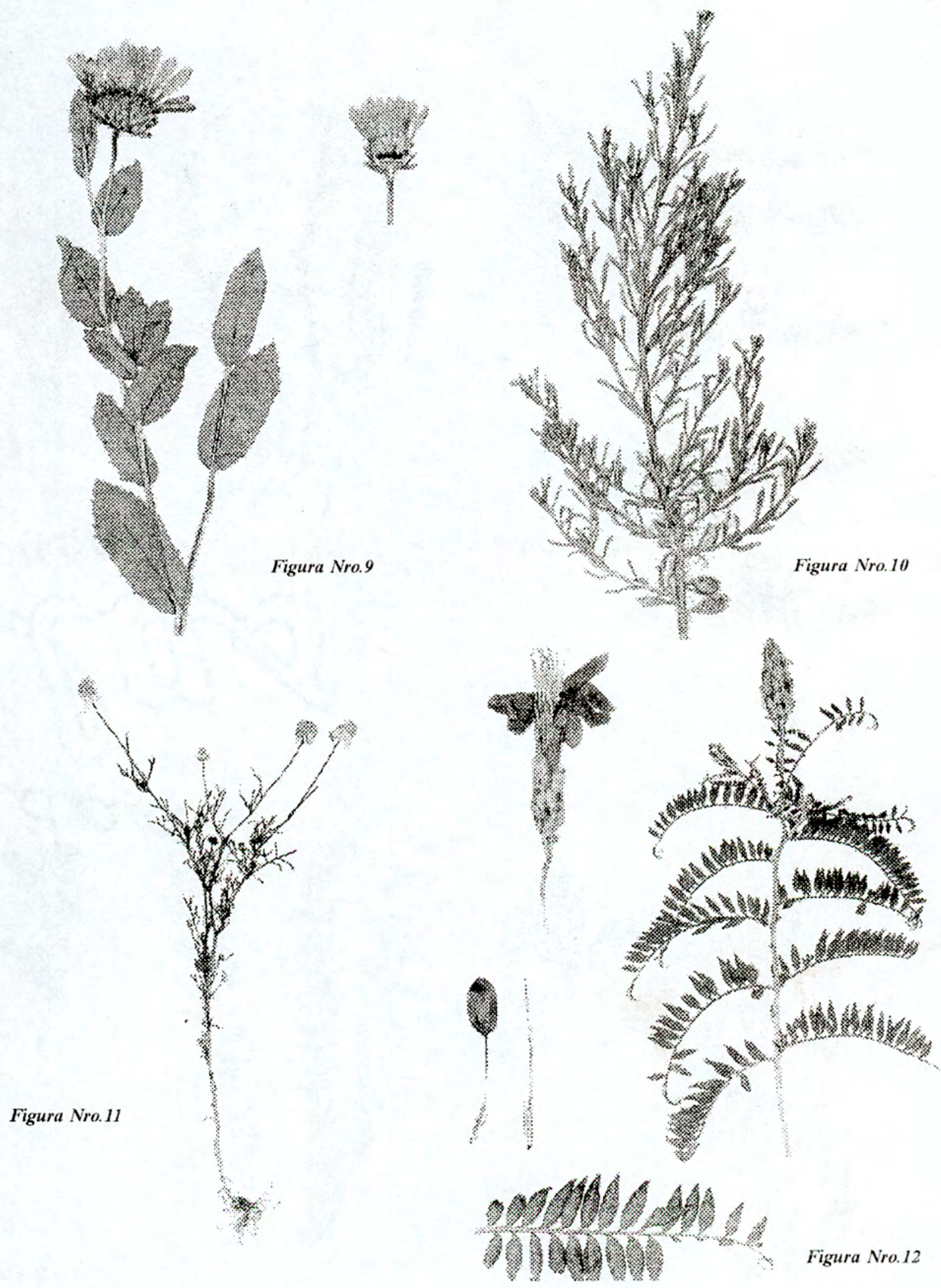

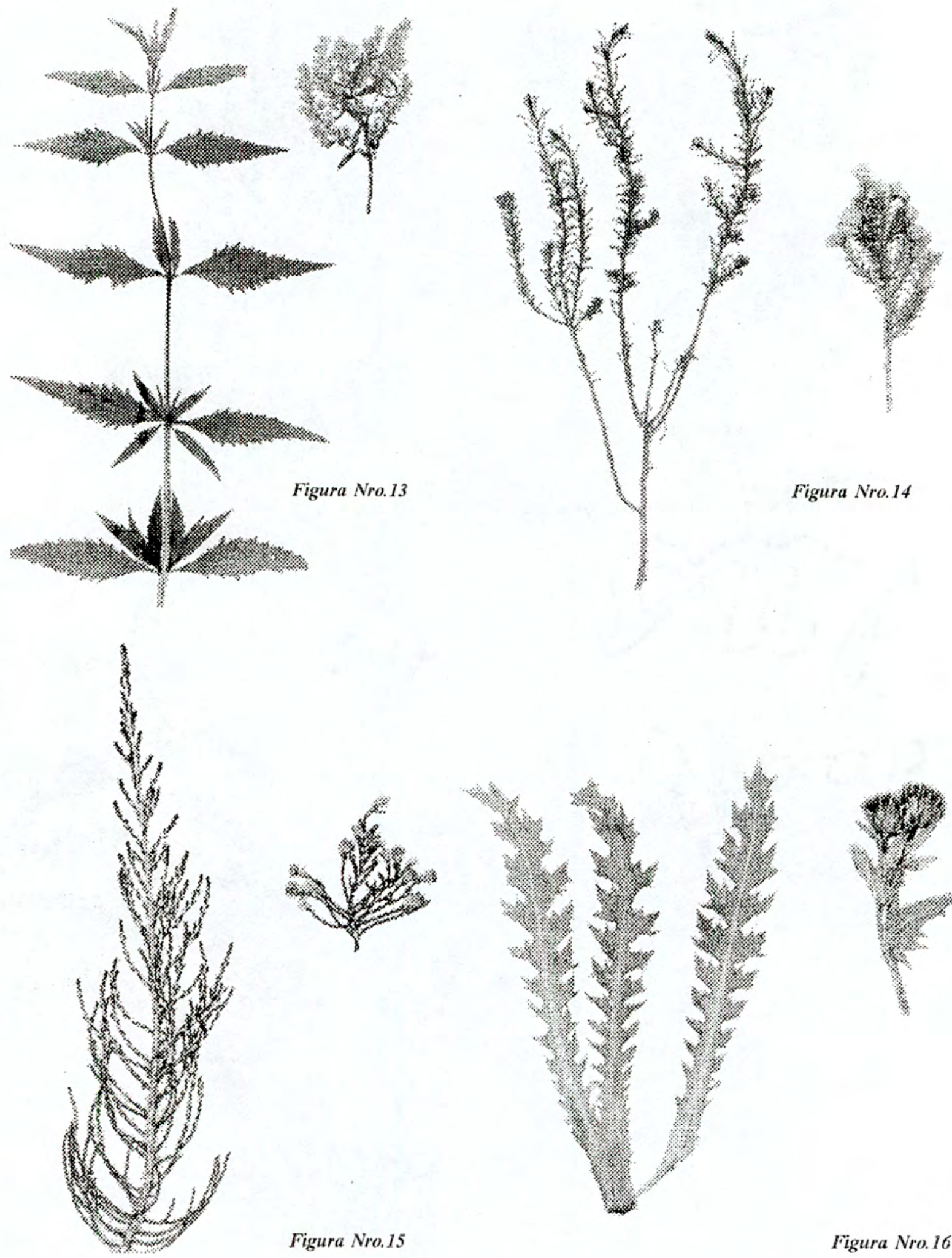

Figura Nro.14

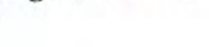



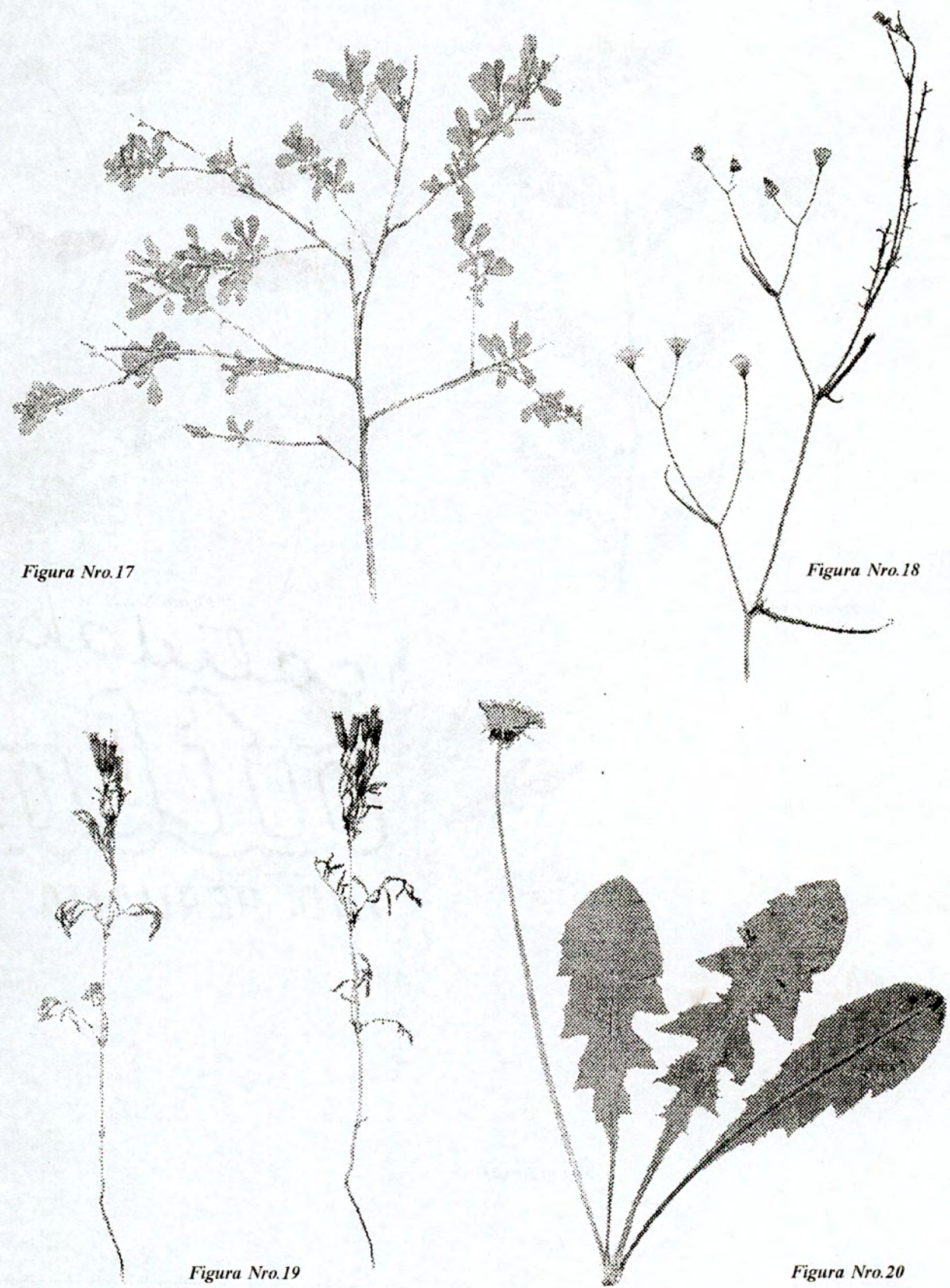


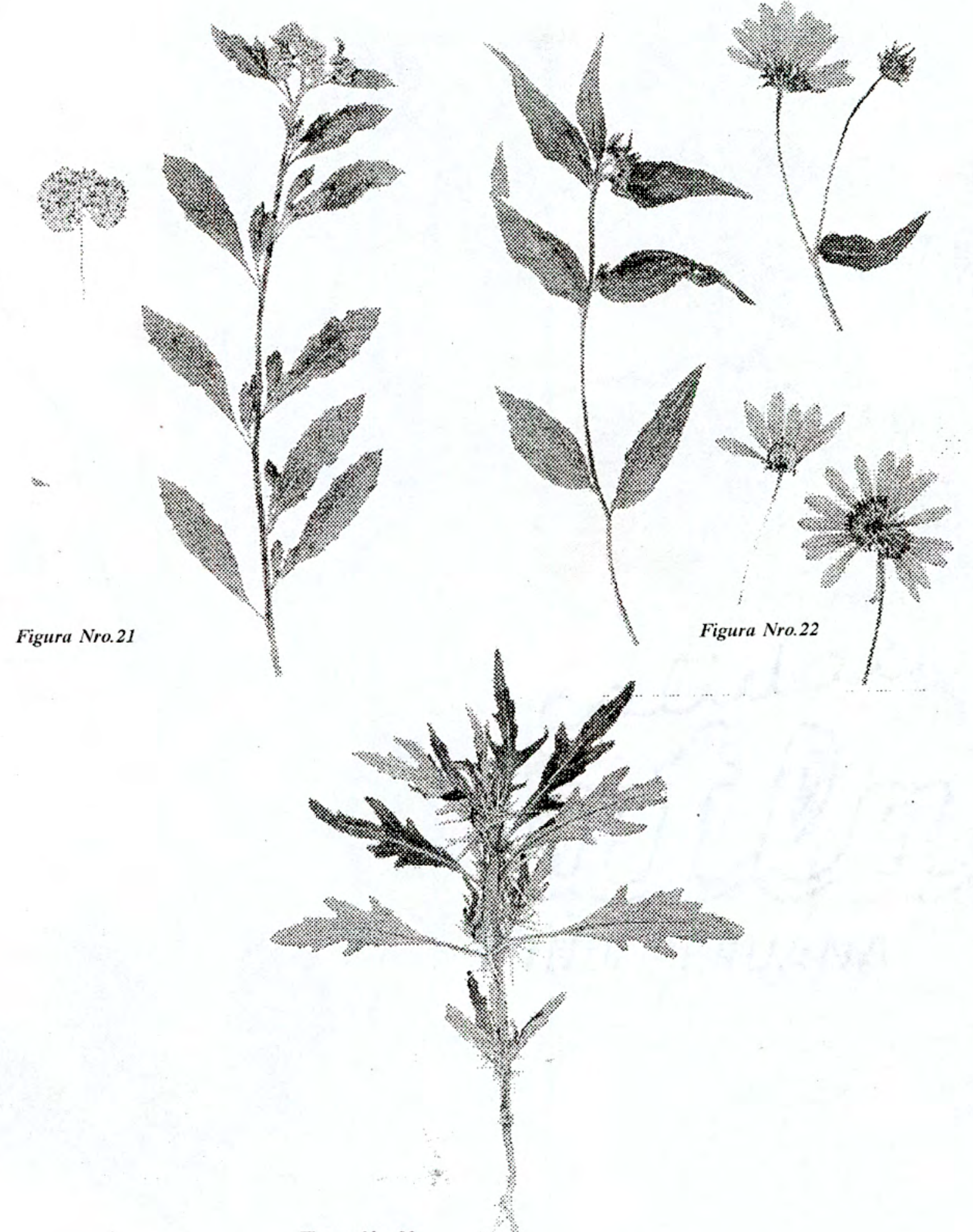

Figura Nro. 23 